\title{
On Nonlinear Systems with Linear Dynamics
}

\author{
Krister Forsman \\ Department of Electrical Engineering \\ Linköping University \\ S-581 83 Linköping, Sweden \\ email: krister@isy.liu.se
}

1994-02-23

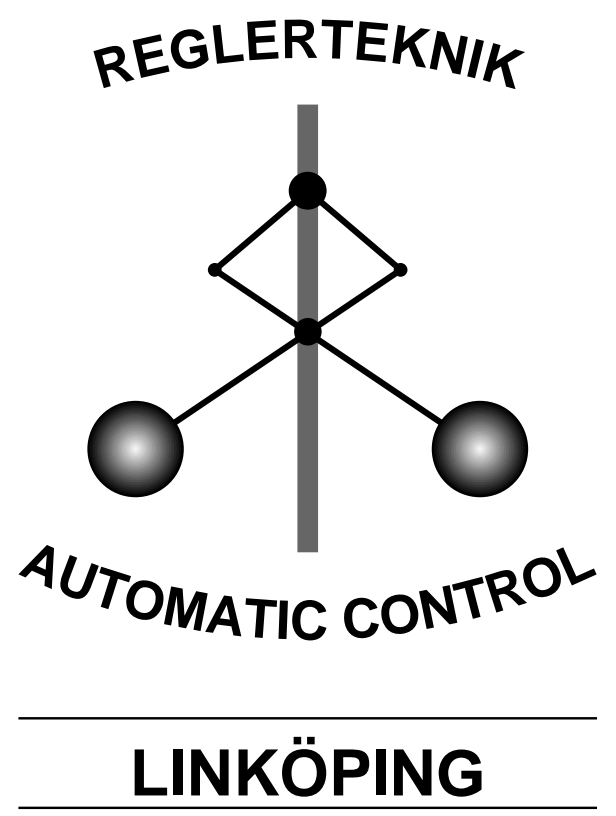

Technical reports from the Automatic Control group in Linköping are available as UNIX-compressed Postscript files by anonymous ftp at the address 130.236.24.1 (joakim.isy.liu.se). 


\title{
On Nonlinear Systems with Linear Dynamics
}

\author{
Krister Forsman \\ Department of Electrical Engineering, Linköping University, \\ S-581 83 Linköping, Sweden \\ Email: krister@isy.liu.se
}

1994-02-23

\begin{abstract}
Some classes of nonlinear systems are investigated and characterized in terms of their input-output behavior. The first class are those that can be realized as a set of linear state equations with a nonlinear output map. The second class are those that can be realized as a set of homogeneous (w.r.t. some grading) state equations with homogeneous output map.
\end{abstract}

Keywords: realization theory, nonlinear control systems, input-output equation, observability, polynomial systems, differential algebra, total degree Gröbner bases

\section{$1 \quad$ Introduction}

It is the purpose of this paper to study a class of nonlinear control systems that are in a sense linear, namely systems that can be written as

$$
\begin{aligned}
& \dot{x}(t)=A x(t)+B u(t) \\
& y(t)=h(x(t))
\end{aligned}
$$

where $h$ is a nonlinear function of $x(t) \in \mathbb{R}^{n}, A$ an $n \times n$ matrix etc. Let us call such systems latently linear. In this paper we will study the differential equation satisified by $y$ and $u$, or, more generally, the differential ideal obtained by eliminating the latent variables $x$. The d.e. for $y$ can be either linear or nonlinear. One of the main questions How does it show on the input-output equation, i.e. the d.e. satsified by $u$ and $y$, if a system can be realized as a latently linear system? An answer to this question is given in sections 2 and 3.

In section 4 we discuss some observability properties of latently linear systems, and in section 5 we will state some results that generalize those concerning latently linear systems to homogeneous systems, i.e. systems such that if $\dot{x}_{i}=f_{i}$, then all terms in the polynomial $f_{i}$ have the same degree.

Latently linear systems are related to nonlinear systems that can be made linear by a nonlinear change of coordinates:

Example 1.1 (Nijmeijer - van der Schaft [11, p. 150])

The system

$$
\begin{aligned}
& \dot{x}_{1}=-x_{1} \ln x_{2} \\
& \dot{x}_{2}=-x_{2} \ln x_{1}+x_{2} u
\end{aligned}
$$


becomes linear in the coordinates $z_{1}=\ln x_{1}, z_{2}=\ln x_{2}$ :

$$
\begin{aligned}
& \dot{z}_{1}=z_{2} \\
& \dot{z}_{2}=u-z_{1}
\end{aligned}
$$

Conditions for the existence of such state transformations are well known, see e.g. [11, chapter 5]. Here we will instead look at systems given in input-output form and ask if the given input-output equation corresponds to a latently linear system. For ease of notation we use subindices to denote time derivatives of the output variable:

$$
y_{i}:=\frac{d^{i}}{d t^{i}} y(t)
$$

Example 1.2 The system

$$
\dot{x}_{1}=-x_{2}, \quad \dot{x}_{2}=x_{1}, \quad y=x_{1}^{2}
$$

has output equation

$$
2 y_{2} y_{0}-y_{1}^{2}+4 y_{0}^{2}=0
$$

This is easily seen by eliminating the state in (5) e.g. using Gröbner bases, as described in $[6,5]$.

The applications of this problem relate to realization theory etc. If we can find a criterion for when a system is latently linear, then we can basically reduce design and analysis problems to those of a linear system.

\section{Characterization via the Input-Output Equation}

We start by considering latently linear systems with an output map that is polynomial in its arguments $x, u$. More general systems can probably be treated, but then the results will be less powerful, especially on the computational side.

The language of differential and commutative algebra is very appropriate for polynomial systems so we will assume some familiarity with terms such as ideal, differential ideal etc. Some references are $[1,2,9,14]$.

A few words on notation: The polynomial ideal generated by $f_{1}, \ldots, f_{N}$ in the polynomial ring $k\left[X_{1}, \ldots, X_{n}\right]$ is denoted $\left\langle f_{1}, \ldots, f_{N}\right\rangle$. The ring of all differential polynomials in the differential indeterminates $X_{1}, \ldots, X_{n}$ is denoted $k\left\{X_{1}, \ldots, X_{n}\right\}$ and the differential ideal generated by $f_{1}, \ldots, f_{N}$ is written $\left[f_{1}, \ldots, f_{N}\right]$. The contraction and extension of an ideal $\mathfrak{a}$ is denoted $\mathfrak{a}^{c}$ and $\mathfrak{a}^{e}$ respectively. For a (possibly differential) ideal $I$ in a (differential) polynomial ring $R$ we write $I: H^{\infty}$ for the contraction of the extension of $I$ to the ring $S^{-1} R$ where $S$ is the multiplicative system $\left\{1, H H^{2}, H^{3}, \ldots\right\}$, where $H$ is a (differential) polynomial. Thus

$$
I: H^{\infty}=I^{e c}=\left(R_{f} I\right) \cap R
$$

This notation, which is rather unfortunate from commutative algebraic point of view, stems from differential algebra $[9,14]$. 
Let $p$ be a differential polynomial in a differential indeterminate $y$. The leader of $p$ is the highest derivative of $y$ occuring in $p$. The initial $I_{p}$ of $p$ is the (unique) polynomial such that

$$
p=I_{p} v^{q}+R(v)
$$

where $v$ is the leader of $p$ and $R(v)$ is a polynomial of degree $<q$ in $v$. The separant of $p$ is $\frac{\partial}{\partial v} p$.

The following theorem gives a necessary condition on the io-equation for a system to be latently linear.

Theorem 2.1 Suppose that the differential polynomial $p$ is of differential order $n$ in $y$ and define $H$ by

$$
H:=\frac{\partial p}{\partial y_{n}} \cdot \operatorname{lcoeff}(p)
$$

If $p=0$ is the input-output equation of a latently linear system then the differential ideal $\Sigma^{c}$ defined by

$$
[p]: H^{\infty}=: \Sigma^{c}
$$

contains a linear element.

Proof. Suppose that $p$ can be realized as

$$
\dot{x}=f(x, u)=A x+b u, \quad y=h(x, u)
$$

The Lie-derivative of $h$ w.r.t. $f$ is $L_{f} h:=\nabla h \cdot f$. We use the notation

$$
h_{i+1}:=L_{f} h_{i}
$$

It is well known [9] that

$$
\Sigma^{c}=\left[\dot{x}_{1}-f_{1}, \ldots, \dot{x}_{n}-f_{n}, y_{0}-h\right] \cap k\{u, y\}
$$

In other words, $\Sigma^{c}$ consists of all polynomial relations between

$$
h_{0}, h_{1}, h_{2}, h_{3}, \ldots \in k\langle u\rangle\left[x_{1}, \ldots, x_{n}\right]
$$

with coefficients from $k\langle u\rangle$. Suppose $h_{0}$ has total degree $d$ in $x$. Since $f$ is affine in $x$, i.e. of total degree $\leq 1$, the total degree of $h_{i+1}$ in $x$ equals the total degree of $h_{i}$ in $x$ for all $i$. There is only a finite number of monomials of degree $\leq d$ in the $n$ variables $x_{1}, \ldots, x_{n}$, viz

$$
\left(\begin{array}{c}
n+d \\
d
\end{array}\right)=: C(n, d)
$$

Thus $h_{0}, \ldots, h_{N}$ are linearly dependent over $k\langle u\rangle$ whenever $N>C(n, d)$, and maybe earlier.

The condition of theorem 2.1 can't be verified algorithmically because we don't know the degree of $h_{0}$. But we have, somewhat stronger than theorem 2.1:

Theorem 2.2 Suppose that the differential polynomial $p$ is of differential order $n$ in $y$. If $p=0$ is the io-equation of a latently linear system with output map of total degree $\leq d$ then the (non-differential) ideal

$$
\left([p]: H^{\infty}\right) \cap K\left[y_{0}, \ldots, y_{C(n, d)}\right]
$$

contains an element which is linear in $y_{0}, \ldots, y_{C(n, d)}$. 
Proof. This follows immediately from the proof of theorem 2.1.

Clearly the necessary condition of theorems 2.1 and 2.2 are not sufficient, for two reasons:

1. An arbitrary nonlinearity in the input $u$ will not affect any of the arguments used in the proof.

2. It is sufficient that $f$ is affine in $x$, i.e. there may be constant terms.

Instead the converse of theorem 2.1 reads as follows: If $\Sigma^{c}$ contains a linear element, then $p$ can be realized as $\dot{x}=f(x, u), y=h(x, u)$ where $f$ is affine in $x$.

\section{Algorithms}

A nice thing with the condition of theorem 2.2 is that it can be checked algorithmically. Let us introduce the abbreviations

$$
\kappa_{N}:=K\left[y_{0}, \ldots, y_{N}\right]
$$

and

$$
\mathfrak{a}_{N}:=\Sigma^{c} \cap \kappa_{N}=\left([p]: H^{\infty}\right) \cap \kappa_{N}
$$

To check theorem 2.2 algorithmically we wish to determine whether the ideal $\mathfrak{a}_{C(n, d)}$ contains a linear element or not. To tackle this question we must first make an observation that explains how differential algebraic problems can be reduced to commutative algebraic ones:

Theorem 3.1 Let $p \in K\{y\}$ be a differential polynomial of differential order $\gamma$ in $y$ and $H$ the product of the separant and initial of $p$. Then for all $i$ we have that

$$
\mathfrak{a}_{i}=\left\langle p, \partial p, \ldots, \partial^{i-\gamma} p\right\rangle: H^{\infty}
$$

Here $\partial=\frac{d}{d t}$.

Proof. Let $f \in K\{y\}$. According to [14, p. 115] we have that

$$
\operatorname{prem}(f, p)=0 \Longleftrightarrow f \in[p]: H^{\infty}
$$

where $\operatorname{prem}(f, p)$ denotes the pseudo-remainder of $f$ w.r.t. $p$. Ritt and Kolchin use the term remainder, but this terminology seems a little old-fashioned; cf. [8]. But the way the pseudoremainder is constructed we realize that (13) is equivalent to

$$
f \in[p]: H^{\infty} \Longleftrightarrow f \in\left\langle p, \partial p, \ldots, \partial^{i-\gamma} p\right\rangle: H^{\infty}
$$

which proves the theorem.

Notice that if we leave out the division by $H$ there is no analog of theorem 3.1. A counterexample, occuring in e.g. [3], is [ $\left.y^{2}\right]$. Also compare with [12, 13].

The next question is how to compute with $I: H^{\infty}$, given a set of generators for an ideal $I$. This is answered by the following theorem:

Theorem 3.2 With notation as above and the ideal $\mathfrak{a}_{C(n, d)}^{\prime}$ in the ring $\kappa_{C(n, d)}[z]$ is defined by

$$
\mathfrak{a}_{C(n, d)}^{\prime}=\left\langle p, \partial p, \ldots, \partial^{C(n, d)-\gamma} p, z H-1\right\rangle
$$

we have that

$$
\mathfrak{a}_{C(n, d)}=\mathfrak{a}_{C(n, d)}^{\prime} \cap \kappa_{C(n, d)}
$$


Proof. This is the well-known Rabinovich trick for computing with $I^{e c}$, where extension and contraction refer to a ring of fractions w.r.t. a multiplicative set generated by some polynomials. For a rather detailed exposition and a proof we refer to [7, theorem 3.2].

Now a computation of a total degree Gröbner basis, see e.g. [2], will tell us if the ideal $\mathfrak{a}_{C(n, d)}$ contains a linear element:

Theorem 3.3 Let $G$ be a Gröbner basis for the ideal $\mathfrak{a}_{C(n, d)}^{\prime}$ defined in theorem 3.2 w.r.t. a total degree term-ordering ranking $z$ higher than all $x$. $G$ contains a differential polynomial which is linear in $y$ iff there is one in $\mathfrak{a}_{C(n, d)}$.

Proof. This is an immediate consequence of the definition of total degree Gröbner basis, once theorems 3.1 and 3.2 have been established.

It is interesting to note the appearance of total degree gb, since the predominant application of gb use lexicographic term orderings.

Example 3.1 The system

$$
\dot{x}_{1}=-x_{2}, \quad \dot{x}_{2}=x_{1}, \quad y=\frac{1}{2} x_{1}^{2}+x_{1}
$$

has the input-output equation

$$
\begin{aligned}
p:= & \left(4 y_{0}^{2}+4 y_{0}+1\right) y_{2}^{2}+\left(-4 y_{1}^{2} y_{0}+16 y_{0}^{3}-2 y_{1}^{2}+24 y_{0}^{2}+12 y_{0}+2\right) y_{2} \\
& -8 y_{1}^{2} y_{0}^{2}-8 y_{1}^{2} y_{0}+y_{1}^{4}-2 y_{1}^{2}+16 y_{0}^{4}+12 y_{0}^{2}+24 y_{0}^{3}+2 y_{0}=0
\end{aligned}
$$

According to theorem 2.2 the differential ideal $\left\{f ; \exists r: H^{r} f \in[p]\right\}$ should contain a linear differential polynomial of order $\leq\left(\begin{array}{l}4 \\ 2\end{array}\right)=6$, and as we compute a total degree gb for $\mathfrak{a}_{5}^{\prime}$ we discover that it contains the polynomial

$$
y_{5}+5 y_{3}+4 y_{1}
$$

A session in the computer algebra system Maple for doing these things looks as follows:

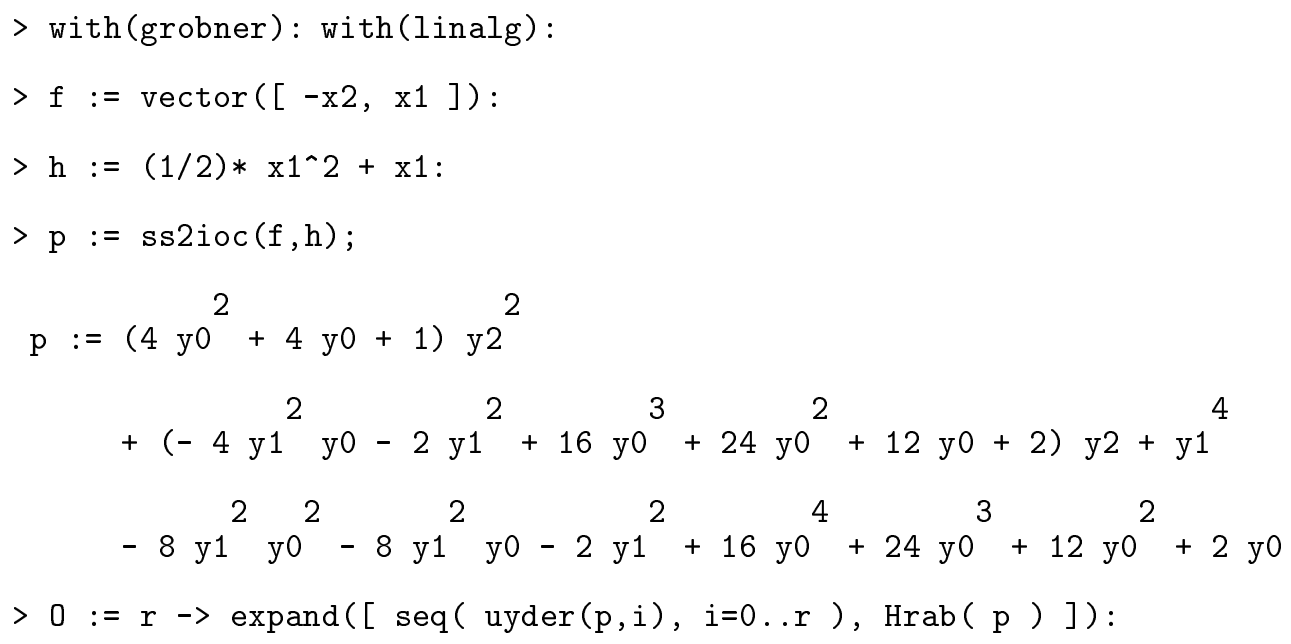




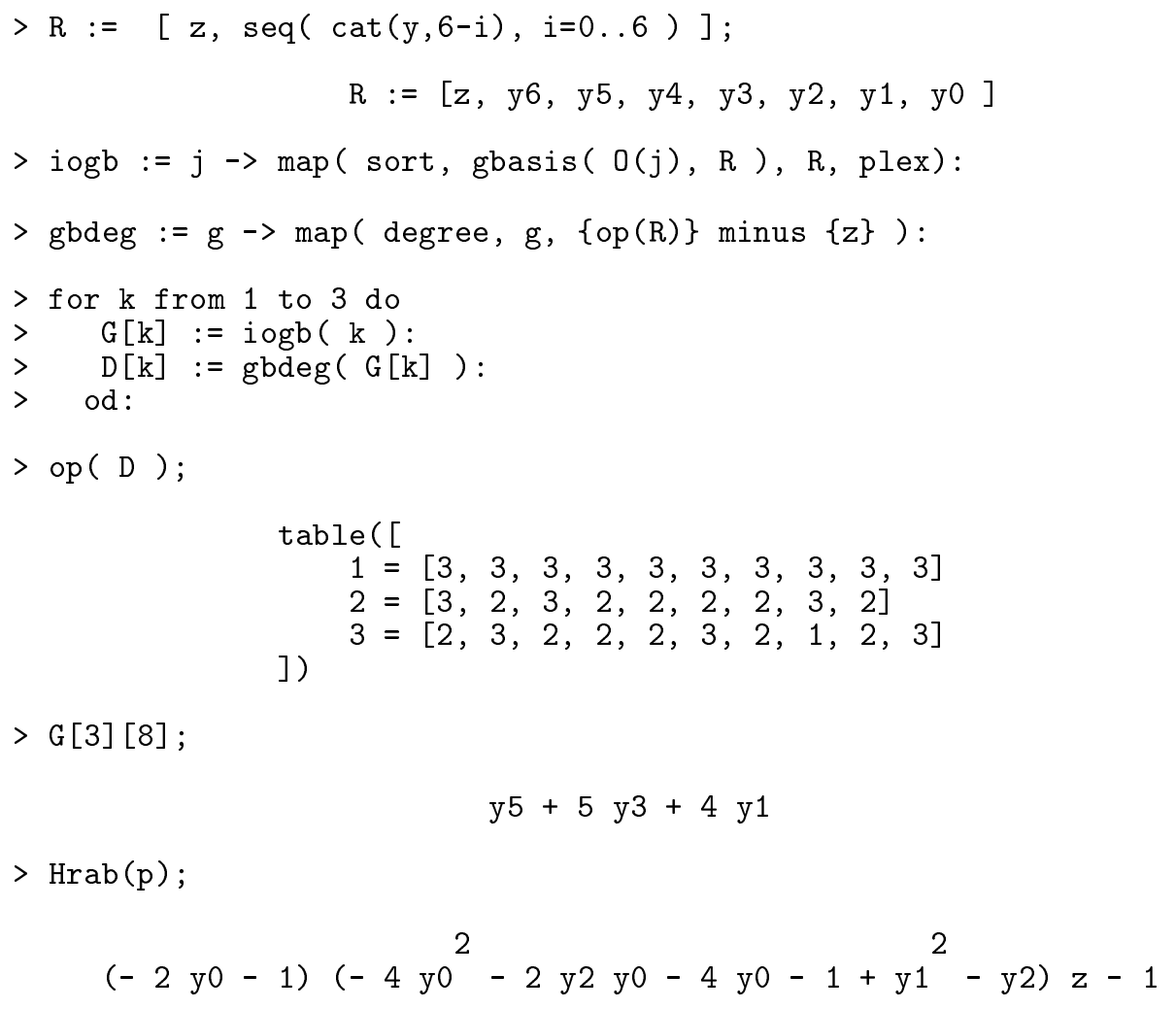

$>\mathrm{G}[3][8]$;

$$
\mathrm{y} 5+5 \mathrm{y} 3+4 \mathrm{y} 1
$$

$>\operatorname{Hrab}(\mathrm{p})$;

$$
(-2 \mathrm{y} 0-1)\left(-4 \mathrm{y}^{2}-2 \mathrm{y} 2 \mathrm{y} 0-4 \mathrm{y} 0-1+\mathrm{y} 1^{2}-\mathrm{y} 2\right) \mathrm{z}-1
$$

Comments:

- The functions ss2ioc and uyder are parts of the POLYCON package, written by the author [5]. The former computes the input-output equation (in this case the scalar output ode) and uyder is just an auxiliary function for differentiating w.r.t. time using indices for time-derivatives of the variables $u, y$.

- The call $\operatorname{Hrab}(\mathrm{p})$ (also implemented by the author) returns the polynomial $z H-1$, where $H$ is a square-free factorization of the product of the initial and the separant of $p$.

\section{Observability and Observers}

In this section we note that observability is particularly simple for latently linear systems.

Theorem 4.1 A latently linear system without inputs is algebraically observable iff its linearization is observable.

Proof. The system $\dot{x}=A x, \quad y=h(x)$ is algebraically observable iff the $n$ polynomials

$$
h_{0}, \ldots, h_{n-1} \in k\left[X_{1}, \ldots, X_{n}\right]
$$

are algebraically independent over $k$. But, as is well known, it follows from the theory Kähler differentials [10, pp. 201-202] that this is the case iff the $n$ vectors

$$
\nabla h_{0}, \ldots, \nabla h_{n-1}
$$


are linearly independent over $k$. The linearized system

$$
\dot{x}=A x, \quad y=\nabla h=C x
$$

is observable iff the observability matrix has full rank, i.e. the $n$ vectors

$$
c, c A, \ldots, c A^{n-1}
$$

are linearly independent over $k$. But since $f=A x$ we have that

$$
h_{i+1}=\nabla h_{i} \cdot A
$$

so $h_{i}=c A^{i}$. This finishes the proof.

It is not difficult to extend theorem 4.1 to systems with inputs, though some additional notational complexity is introduced. (Basically, $k$ has to be replaced by $k\langle u\rangle$.)

The design of observers is also easier for latently linear systems than for a general nonlinear system. We don't enter into a discussion of that at this stage due to limited space. It seems that this result has been used earlier in the literature, but the author has not been able to find a reference to an article describing this.

\section{$5 \quad$ Homogeneous Systems}

We start this section by recalling a few facts about gradings of polynomial rings. The exposition will be short and informal; a more complete treatment is given in [10].

A grading of a polynomial ring is a generalization of the degree of a monomial. Requirements:

$$
\operatorname{deg}(\text { constant })=0, \quad \operatorname{deg}(p q)=\operatorname{deg}(p)+\operatorname{deg}(q)
$$

The value of deg is most often an integer $\mathbb{N}^{n}$-valued degrees occur. A grading is completely determined by the degrees of the generators of the ring, i.e. the variables.

\section{Example 5.1 If}

$$
\operatorname{deg}\left(x_{1}\right)=4, \operatorname{deg}\left(x_{2}\right)=1, \operatorname{deg}\left(x_{3}\right)=0
$$

then $\operatorname{deg}\left(x_{1}^{2} x_{2}^{3} x_{3}\right)=11$

A polynomial is homogeneous w.r.t. a given grading if all its terms have the same degree. E.g. the polynomial

$$
x_{1}-3 x_{2}^{4}+x_{3} x_{1}
$$

is homogeneous w.r.t. the grading in example 5.1. The grading given by $\operatorname{deg}\left(x_{i}\right) \equiv 1$ is referred to as the standard grading.

An ideal $I$ in $k\left[X_{1}, \ldots, X_{n}\right]$ is homogeneous if it can be generated by a set of homogeneous polynomials (not necessarily of the same degree).

Note: this does not mean that all elements of $I$ are homogeneous.

Theorem 5.1 $I$ is homogeneous iff for every $p \in I$ written $p=\sum p_{i}$ where $p_{i}$ is homogeneous of degree $i$, we have that $p_{i} \in I$. 
Proof. Standard exercise in algebraic geometry. See e.g. [16].

We will need a number of properties of homogeneous polynomials, that are more or less well known.We recall these auxiliary theorems here. Their proofs are easy. Assume that $G$ is a grading of a polynomial ring $R$.

Theorem 5.2 Let $\partial$ be an arbitrary derivation on $R$ and $p \in R$ a $G$-homogeneous polynomial. Then $\partial p$ is also $G$-homogeneous.

Theorem 5.3 If $p \in R$ is $G$-homogeneous then the separant of $p$ is $G$-homogeneous.

Proof. Choose $\partial=\frac{\partial}{\partial v}$ in theorem 5.2, where $v$ is the leader of $p$.

Theorem $5.4 a, b \in R$ are $G$-homogeneous iff their product ab is $G$-homogeneous.

Theorem 5.5 If $p$ is G-homogeneous then so is the initial of $p$.

Proof. The initial $I_{p}$ is such that $p=I_{p} v^{q}+R(v)$ If $p$ is $G$-homogeneous, then so is $I_{p} v^{q}$, so it follows from theorem 5.4 that $I_{p}$ is $G$-homogeneous.

Lemma 5.1 If some polynomials $p_{1}, \ldots, p_{N}$ are homogeneous of degrees $\alpha_{1}, \ldots, \alpha_{N}$ w.r.t. some grading, then the ideal of all relations between the $p_{i}$ 's (the syzygy ideal) is homogeneous w.r.t. the grading $\operatorname{deg}\left(p_{i}\right) \equiv \alpha_{i}$.

Definition 5.1 A differential ideal $\mathfrak{k}$ is differentially homogeneous if there is a non-differential homogeneous ideal $I$ (not finitely generated, of course) such that $\mathfrak{k}=I$. (This definition may be non-standard.)

Now, let $K:=k\langle u\rangle$ and let $K[x]_{d}$ denote the $K$-space of all polynomials of total degree $d$ in the variables $x$ (coefficients from $K$ ). Thus $K[x]_{d}=\{$ all polynomials homogeneous of degree $d$ w.r.t. the standard grading $\}$. We will consider systems

$$
\dot{x}=f(x, u), \quad y=h(x, u)
$$

where $f_{1}, \ldots, f_{n}, h \in K[x]$.

We will now state two theorems that characterize the input-output differential ideal of homogeneous systems. For pedagogical reasons the less general one comes first.

Theorem 5.6 If all nonzero $f_{i} \in K[x]_{\alpha}$ and $\left.h \in K\right]_{\beta}$ for some $\alpha, \beta \in \mathbb{N}$ then the ideal $\Sigma^{c}$ is differentially homogeneous w.r.t. a grading

$$
G_{1}: \quad \operatorname{deg}\left(y_{j}\right)=(\alpha-1) \cdot j+\beta
$$

i.e. $\Sigma^{c}$ is non-differentially generated by an (infinite) set of polynomials that are $G_{1}$-homogeneous.

Proof. If $f_{i}$ are homogeneous of degree $\alpha$ and $h_{0}$ is homogeneous of degree $\beta$ then

$$
h_{j+1}=\sum_{i} f_{i} \frac{\partial}{\partial x_{i}} h_{j}
$$


will be homogeneous of degree

$$
\operatorname{deg}\left(h_{j}\right)-1+\alpha
$$

By recursion we get that

$$
h_{j} \in K[x]_{j(\alpha-1)+\beta}
$$

Since $\Sigma^{c}$ is the syzygy ideal of $h_{0}, h_{1}, h_{2}, h_{3}, \ldots$ in $K\{y\}$ we only have a special case of lemma 5.1.

Example 5.2 The input-output ideal of the system

$$
\dot{x}_{1}=x_{1} x_{2}, \quad \dot{x}_{2}=u x_{1}^{2}, \quad y=x_{1}^{3}
$$

is differentially homogeneous w.r.t. the grading

$$
\operatorname{deg}\left(y_{j}\right)=j+3, \quad \operatorname{deg}\left(u_{i}\right) \equiv 0
$$

E.g. the input-output equation is

$$
y_{2}^{3} y_{0}^{3}-3 y_{2}^{2} y_{1}^{2} y_{0}^{2}+3 y_{2} y_{1}^{4} y_{0}-y_{1}^{6}-27 u_{0}^{3} y_{0}^{8}
$$

Now we have the following generalization of theorem 5.6:

Theorem 5.7 Consider a grading $G_{1}$ given by $\operatorname{deg}_{1}\left(x_{i}\right) \equiv \delta_{i}$ and a system

$$
\dot{x}=f(x, u), \quad y=h(x, u)
$$

If $h$ and all $f_{i}$ are $G_{1}$-homogeneous and

$$
\operatorname{deg}_{1}\left(f_{1}\right)-\delta_{1}=\ldots=\operatorname{deg}_{1}\left(f_{n}\right)-\delta_{n}=\mu
$$

then $\Sigma^{c}$ is differentially homogeneous w.r.t. the grading

$$
G_{2}: \quad \operatorname{deg}_{2}\left(y_{j}\right)=\mu \cdot j+\beta
$$

where $\beta=\operatorname{deg}_{1}(h)$

Proof. The key idea is to ask when the Lie-derivative operator preserves homogeneity. The answer is that it is necessary and sufficient that $\operatorname{deg}\left(f_{i}\right)-\operatorname{deg}\left(x_{i}\right)$ is constant in $i$. The proof of this is very straightforward, so we don't reproduce it here.

Remark: Theorem 5.6 is the special case $\delta_{i} \equiv 1, \mu=\alpha-1$.

To make theorem 5.7 more useful we need a characterization of when $\Sigma^{c}$ is differentially homogeneous. The following theorem does the job:

Theorem $5.8 \Sigma^{c}$ differentially homogeneous w.r.t. a permissible grading G iff $p$ is. 
Proof. According to lemma 5.2 the differential ideal $[p]$ is differentially homogeneous w.r.t. $G$, i.e. the polynomial ideal

$$
I:=\left\langle p, \partial p, \partial^{2} p, \ldots\right\rangle
$$

in the (non-differential) ring $K\left[y_{0}, y_{1}, y_{2}, \ldots\right]$ is homogeneous. If we can prove that

$$
I^{e c}:=(I+\langle z H-1\rangle) \cap K\{y\}
$$

is again differentially $G$-homogeneous we are done. It follows from theorems 5.3 and 5.5 that $H$ is homogeneous. Pick an arbitrary element $f \in I^{e c}$ and write it as a sum of its homogeneous components:

$$
f=\sum f_{i}
$$

According to theorem 5.1 above, $I^{e c}$ is homogeneous iff all $f_{i} \in I^{e c}$. But if $f=H g$ for some $g \in I$ then $f_{i}=H^{t} g_{j}$ for some $j, t \in \mathbb{N}$ and $g_{j} \in I$ so $f_{i} \in I^{e c}$

We conclude by a comparison with the Kolchin permissible gradings of differential algebra [9, page 72]. First recall a few basic facts:

Definition 5.2 A permissible grading of an ordinary differential polynomial ring is a grading such that for every differential indeterminate $u$

$$
\operatorname{deg}\left(\partial^{e} u\right)=\mu \cdot e+\nu_{u}
$$

for some $\mu, \nu_{u} \in \mathbb{N}$.

Lemma 5.2 A permissible grading $G$ has the property that the derivative of a $G$-homogeneous differential polynomial $f$ is again homogeneous but of degree $\mu+\operatorname{deg}(f)$.

Proof. That $f$ is homogeneous follows directly from theorem 5.2.

A well known special case of permissible gradings is $\mu=0, \nu=1 \Rightarrow$ standard grading.

Clearly all systems discussed in 5.6 have input-output ideals that are homogeneous w.r.t. a permissible grading.

\section{Open Problems}

Some interesting open problems relating to homogeneous and latently linear systems are:

- Is the bound in theorem 2.2 sharp?

- Are there any connections between theorem 5.7 and the unirationality of projective hypersurface defined by the input-output equation? In [4] it was proved that rationality issues in algebraic geometry are very closely related to realizability of differential equations.

- Does the Volterra series appraoch to nonlinear systems (see e.g. [15]) shed more light on latently linear systems?

- Is the condition in theorem 5.6 sufficient? 
- If $p$ is the output map of a latently linear system, can an upper bound for the degree of the output map be established using only $p$ ? In that case theorems 2.2 and 3.3 together provide a complete algorithm for testing if a given nonlinear ode can be realized as a latently linear system.

- What kind of generalizations are possible to non-polynomial nonlinearities?

\section{Acknowledgement}

This work was financially supported by the Swedish Council for Technical Research (TFR).

\section{References}

[1] M.F. Atiyah and I.G. MacDonald. Introduction to Commutative Algebra. AddisonWesley, 1969.

[2] D. Cox, J. Little, and D. O'Shea. Ideals, Varieties, and Algorithms: An Introduction to Computational Algebraic Geometry and Commutative Algebra. Undergraduate Texts in Mathematics. Springer, 1992.

[3] G. Carrà Ferro. Gröbner bases and differential algebra. In L. Huguet and A. Poli, editors, Applied Algebra, Algebraic Algorithms and Error-Correcting Codes, volume 356 of Lecture Notes Comp. Sci., pages 129-140. Springer, 1989. Proc. AAECC-5, Menorca.

[4] K. Forsman. On rational state space realizations. In M. Fliess, editor, Proc. NOLCOS'92, pages 197-202, Bordeaux, 1992. IFAC.

[5] K. Forsman. POLYCON - computer algebra software for polynomial control systems. In Proc. 32:nd Conf. on Decision and Control, volume 1, pages 282-287, San Antonio, Texas, 1993. IEEE CSS.

[6] K. Forsman. Some generic results on algebraic observability and connections with realization theory. In J.W. Nieuwenhuis, C. Praagman, and H.L. Trentelman, editors, Proc. 2nd European Control Conf., volume 3, pages 1185-1190, Groningen, July 1993.

[7] P. Gianni, B. Trager, and G. Zacharias. Gröbner bases and primary decomposition of polynomial ideals. In L. Robbiano, editor, Computational Aspects of Commutative Algebra, pages 15-33. Academic Press, 1989. From J. Symbolic Computation Vol. 6, nr. 2-3.

[8] D.E. Knuth. The Art of Computer Programming. Vol. 2: Seminumerical Algorithms. Addison-Wesley, second edition, 1981.

[9] E.R. Kolchin. Differential Algebra and Algebraic Groups., volume 54 of Pure and Applied Mathematics. Academic Press, 1973.

[10] H. Matsumura. Commutative Ring Theory., volume 8 of Cambridge Studies in Advanced Mathematics. Cambridge University Press, 1986. 
[11] H. Nijmeijer and A.J. van der Schaft. Nonlinear Dynamical Control Systems. Springer, 1990 .

[12] F. Ollivier. Standard bases of differential ideals. In S. Sakata, editor, Applied Algebra, Algebraic Algorithms and Error-Correcting Codes, volume 508 of Lecture Notes Comp. Sci., pages 304-321. Springer, 1990. Proc. AAECC-8, Tokyo.

[13] F. Ollivier. Generalized standard bases with applications to control. In Proc. First European Control Conf., volume 1, pages 170-176, Grenoble, France, July 1991. Hermès.

[14] J.F. Ritt. Differential Algebra. Dover, 1950.

[15] M. Schetzen. The Volterra and Wiener Theories of Nonlinear Systems. Wiley, 1980.

[16] I.R. Shafarevich. Basic Algebraic Geometry. Springer, study edition, 1977. 